Original Research Paper

\title{
Conservation of Binding of Llama and Other Animals' Hemoglobins to Haptoglobins Across Species
}

\author{
${ }^{1}$ Moges Woldemeskel and ${ }^{2}$ Oluseyi A. Vanderpuye \\ ${ }^{1}$ Department of Pathology, College of Veterinary Medicine, \\ Tifton Veterinary Diagnostic and Investigational Laboratory, University of Georgia, 43 Brighton Rd, Tifton, GA USA \\ ${ }^{2}$ Department of Chemistry and Forensic Science, Albany State University, Albany, GA USA
}

Article history

Received: 10-10-2017

Revised: 05-01-2018

Accepted: 25-01-2018

Corresponding Author: Oluseyi A. Vanderpuye Department of Chemistry and Forensic Science, Albany State University, Albany, GA USA Email:seyi.vanderpuye@asurams.edu

\begin{abstract}
The plasma protein haptoglobin binds hemoglobin released from lysed erythrocytes. It causes removal of the free hemoglobin, thus preventing pathological oxidation of cells. Hemoglobin higher level structures are well conserved across animal species but primary structures can be as little as $50 \%$ homologous. Because of these differences, the question arose as to what extent hemoglobins can bind to haptoglobins from different species. The charge properties and binding to three genetic variants of human haptoglobin were compared by non-denaturing agarose gel electrophoresis, with llama, human, dog, pig, horse and goat hemoglobins. In this study, it was reported for the first time that llama and alpaca hemoglobins differed in electrophoretic mobility from hemoglobins from several animal species and humans. Llama hemoglobin was more positively charged than the other mammalian hemoglobins. Electrophoretic mobility changes of the animal hemoglobins in the presence of human plasma and two different purified human haptoglobin genetic variants suggested that hemoglobins from all animals in this study could bind all three genetic variants of human haptoglobin. In all cases, the llama hemoglobin-haptoglobin samples had lesser mobility than those of the other mammals. This study showed that the binding sites on hemoglobin and haptoglobin for each other have been evolutionarily conserved despite differences in primary structure and marked difference in the charge of llama hemoglobin from the other animal species and humans.
\end{abstract}

Keywords: Blood, Erythrocytes, Haptoglobin, Hemoglobin, Llama, Proteins, Polymorphism 1

\section{Introduction}

Hemoglobin is essential in vertebrates as anoxygen transport protein but when released from lysed erythrocytes can damage cells and tissues and especially kidneys by oxidative processes. A major mechanism for prevention of hemoglobin-mediated injury involves the plasma protein haptoglobin which is synthesized in the liver (Quaye, 2008; Shaer et al., 2014) (Fig. 1). Haptoglobin has an extremely high affinity $\left(\mathrm{Ka} \sim 10^{15}\right)$ for hemoglobin and its binding leads to decreases in hemoglobin mediated damage to kidneys (Quaye, 2008). The hemoglobin-haptoglobin complex is removed from blood by binding to the macrophage cell surface protein CD163 followed by endocytosis (Quaye, 2008; Shaer et al., 2014) (Fig. 1).

Hemoglobin varies among animal species in its sequence, its oxygen-binding and its regulation by small molecules (Perutz, 1983). The structural variation in hemoglobins and increased affinity for oxygen have been correlated with such factors as species habitats and lifestyles such as high altitude environments for llama, guinea pigs and other Andean species (Perutz, 1983; Jessen et al., 1991; Storz, 2007; Storz and Moriyama, 2008; Painet and Jaenicke, 2010). Increased affinity for oxygen for some hemoglobins is also correlated with high altitude flight for vultures and black headed gull, bar headed goose and Andean geese (Hieblet al., 1988; Godovac-Zimmermann et al., 1988; Jessen et al., 1991). In other cases, lowered affinity of hemoglobin for oxygen is correlated with certain amino acid sequence substitutions and cold habitats such as for Antarctic penguins and fish (Notothenoide) and woolly mammoths (Stam et al., 1997; diPrisco et al., 2000; Campbell et al., 2010). 


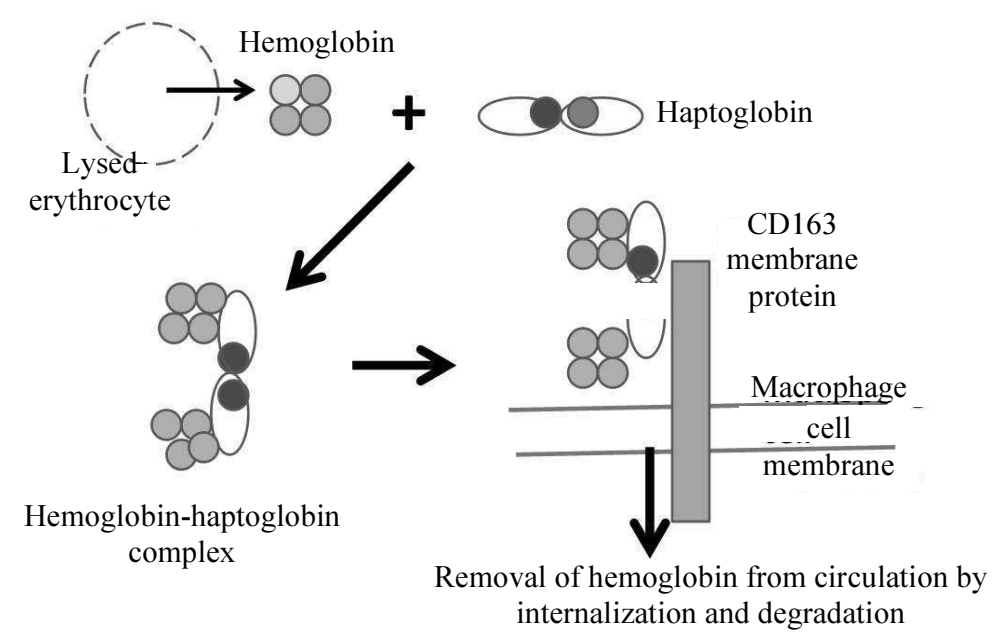

Fig. 1: Haptoglobin functions in removal of extracellular hemoglobin: Hemoglobin released from lysed erythrocytes into blood binds to the protein haptoglobin. The complex of hemoglobin and haptoglobin binds to the cell surface integral membrane protein CD163 on macrophages. CD163 mediates endocytosis of the hemoglobin-haptoglobin complex into macrophages

Diving mammals such as seals, sea lions and walruses also express hemoglobins with sequence differences in comparison to other mammals (Watanabe et al., 1986; Lin et al., 1989; Ikehara et al., 1996). However, in an extreme example, certain Antarctic fish genetically do not express hemoglobin which is a condition not described in other vertebrates (Cocca et al., 1995).

In the case of the llama, its hemoglobin differs biochemically from those of many other mammals living at lower altitudes. Llama hemoglobin is able to bind oxygen more efficiently at low oxygen concentrations and responds differently to 2-diphosphoglycerate than some other species such as human (Piccinini et al., 1990; Storz, 2007).

It has been repeatedly found that hemoglobins from phylogenetically separated species or groups generally differ more in amino acid sequence than do hemoglobins from phylogenetically similar species which are more similar in amino acid sequence to each other. Therefore, based on extensive studies, there is good evidence for interspecies differences in hemoglobin amino acid sequences and how these affect the properties of hemoglobin. The interspecies differences in hemoglobin amino acid sequence suggest the question of whether hemoglobin interactions with other proteins vary among animal species.

Haptoglobin consists of two polypeptide chains which are a smaller alpha subunit and a larger beta subunit. These polypeptides are usually linked by disulfide bonds in a basic tetrameric (alpha chain -beta chain)2 arrangement (Fig. 2). In most species, the alphabeta units are usually but not always linked by disulfide bonds. Haptoglobin also shows interspecies biochemical differences. Human haptoglobin has a genetic polymorphism that is absent from other mammals including primates (Quaye, 2008; Wobeto et al., 2008; Campbell et al., 2010; Shaer et al., 2014). It exists as Hp 1:1, 2:1 and 2:2 variants (Fig. 2). Ruminant haptoglobins such as those from cattle, goat, sheep, deer and water buffalo differ from those of other mammals by existing in a multimeric form. The haptoglobins of these species are different from those of other mammals but similar to the human Hp 2:2 allele in having a partial tandem duplication of sequence in the haptoglobin alpha chain (Busby and Travis,1978; Lai et al., 2007; Wicher and Fries, 2007; Lai et al., 2008; Wicher and Fries, 2010; Niranjan et al., 2015). Haptoglobins from carnivores have their own structural distinctions from other mammalian haptoglobins. The haptoglobin alpha-beta subunit pairs of dog, bear and cat are linked noncovalently into tetramers. There are no disulfide bonds between the alpha subunits in the tetramer unlike in haptoglobins of other species. The dog alpha chain also has N-linked oligosaccharides unlike those of other mammals (Kumazaki et al., 1992; Mominoki et al., 1995). The amino acid sequence of dog haptoglobin alpha chain is $68 \%$ identical to human haptoglobin alpha chain and the respective beta subunits are $85 \%$ identical in amino acid sequence (Kumazaki et al., 1992).

The entries in the UniProt sequence database provide much evidence of sequence differences among haptoglobins that often but not always group according to phylogenetic classifications. For example, the Sambar deer (Rusa unicolor swinhoel) haptoglobin is $98.2 \%$ identical in sequence to another deer Cervus elaphus, 91.4\% identical to cattle and water buffalo haptoglobins but shows lower sequence identity to haptoglobins fromhuman (75.5\%), gorilla (72.95), mouse $(73.2 \%)$ and rat $(72.6 \%)$. Haptoglobins also differ among species in that these proteins have been found in fish and mammals and ostriches but are absent from chicken and the frog Xenopus. In chickens another hemoglobin binding protein PIT54 is present in blood. Ostrich blood contains both PIT54 and haptoglobin (Wicher and Fries, 2006). 


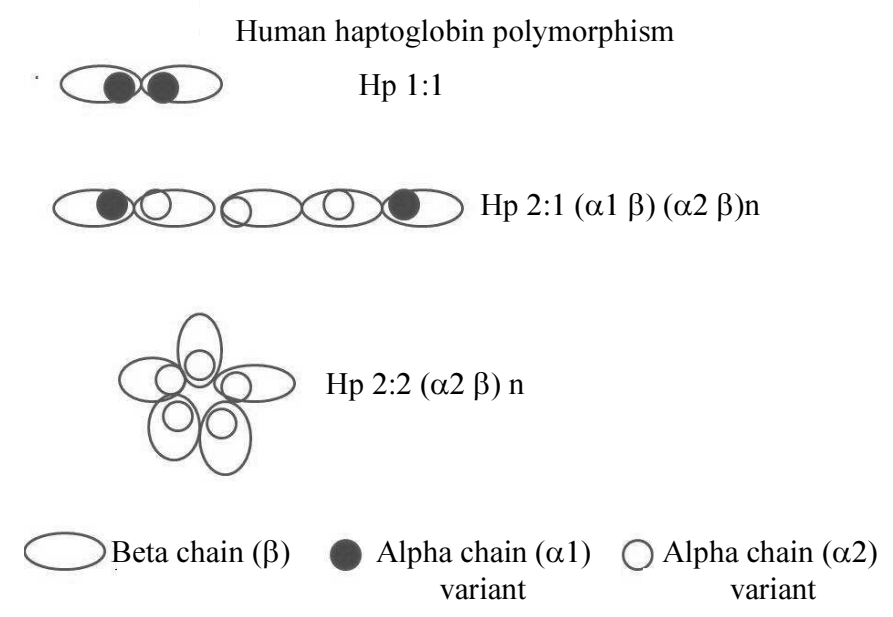

Fig. 2: Human haptoglobin exists as three genetic variants that differ in oligomeric arrangement: Human haptoglobin variants are depicted. Haptoglobin 1:1 (Hp 1:1) consists of two $\beta$ chains and two smaller $\alpha$ chains and is a tetramer $(\alpha 1 \beta)_{2}$. Haptoglobin 2:1 (Hp2:1) expresses the $\alpha 1$ polypeptide and the $\alpha 2$ polypeptide and the $\beta$ chain. Haptoglobin 2:1 consists of the tetramer found in Hp 1:1 $(\alpha 1 \beta)_{2}$ and alsohigher orderlinearoligomers $(\alpha 2 \beta) \mathrm{n}$. Only one example is diagrammed. Haptoglobin 2:2 consists only of higher order oligomers of $(\alpha 2 \beta)$ but these are more compactly or even circularly arranged in contrast to Hp 2:1

Differences in immunochemical reactivities provide other evidence for similarities and differences in haptoglobin structure among species. A mouse monoclonal antibody to human haptoglobin recognized goat, sheep and bovine haptoglobins and to a lesser extent horse and rabbit haptoglobins. This antibody did not react with haptoglobins from dog, fox, cat and pig (Katnik et al., 1998). Another study found that an antibody preparation reacted with goat, sheep and bovine haptoglobins but not with pig haptoglobin (Busby and Travis, 1978). A commercial immunoturbidometric assay for human haptoglobin reacted with the dog protein and another polyclonal antibody to human haptoglobin reacted with the dog protein in ELISA and immunodiffusion assays (Tecles et al., 2007). The immunochemical results indicate that haptoglobins vary in structural details among different animal species.

Because both hemoglobin and haptoglobin from different species differ structurally, the question arises as to the extent to which hemoglobins and haptoglobins from different species can bind each other.

Two opposing hypotheses can be made. (1) Haptoglobin binding to hemoglobin is of fundamental physiological importance and once evolved, the protein structural elements involved have been conserved among vertebrate hemoglobins and haptoglobins although other sites on these molecules have changed during the evolution of different species.

(2) The evolutionary changes in haptoglobin and hemoglobin sequences have affected the abilities of haptoglobins from some species to bind to hemoglobins from other species.
The objectives of this study were to examine: (1) charge differences of different animal hemoglobins by electrophoresis (2) binding of hemoglobins from different species to the plasma protein haptoglobin in human and horse plasmas (3) binding of hemoglobins from different animal species to human haptoglobin genetic variants $\mathrm{Hp} 1: 1$ and $\mathrm{Hp} 2: 2$ (4) isolation of haptoglobin fromplasmas of different animal species by affinity chromatography with llama hemoglobinSepharose conjugate.

\section{Materials and Methods}

\section{Materials}

Llama, canine, bovine, goat, equine and porcine whole blood samples were obtained from Lampire Biological Laboratories, Pipersville, PA, USA. Llama, feline and anteater blood samples were provided by Dr. Woldemeskel, University of Georgia, Veterinary Diagnostic and Investigational Laboratory, Tifton, GA, USA. Whole human blood samples were obtained from Zen Bio Inc., Research Triangle Park, NC, USA. FicollPaque Plus (Ficoll) was purchased from GE Healthcare Bio-Sciences, Pittsburgh, PA, USA. Cyanogen bromideactivated Sepharose CL-4B and other biochemical reagents were obtained from Sigma Chemicals, St. Louis MO, USA. Agarose, Tris-Glycine pH 8.3 electrophoresis buffer, Kaleidoscope Precision Plus pre-stained molecular weight standards, nitrocellulose and other electrophoresis materials were obtained from Biorad Laboratories, Hercules, CA, USA. The Bicinchoninic Acid (BCA) protein concentration determination kit was 
purchased from Pierce, Rockford, IL, USA. Human haptoglobin genetic variants $\mathrm{Hp} \mathrm{1:1} \mathrm{and} \mathrm{Hp} \mathrm{2:2} \mathrm{were}$ obtained from Lee Biosolutions, Maryland Heights, MO, USA. The human hemoglobin lateral flow immunochromatographic assay was purchased from Serological Research Institute, Richmond, CA, USA.

\section{Methods}

\section{Isolation of Erythrocytes and Plasma from Whole Blood}

In order to isolate fractions containing plasma and erythrocytes, acid citrate dextrose anti-coagulated whole blood was processed by centrifugation through layers of Ficollby adapting the manufacturer's instructions (GE Healthcare manual:Instructions 71-7167$00 \mathrm{AG})$.Volumes of 2 to $4 \mathrm{~mL}$ of whole blood were layered onto $3 \mathrm{~mL}$ of Ficoll in $15 \mathrm{~mL}$ conical centrifuge tubes and centrifuged in a bench centrifuge for $8 \mathrm{~min}$. The layer above the Ficoll constituted plasma; and the erythrocytes were found as a pellet below the Ficoll.

The plasma layer was removed by aspiration with a disposable transfer pipette and centrifuged at 14,000 rpm in a Hettich microcentrifuge to sediment platelets and the supernatant was removed and retained as a preparation of plasma. The erythrocytes were retrieved after removal of the Ficoll, resuspended in $10 \mathrm{~mL}$ of Tris Buffered Saline (TBS), sedimented by centrifugation and washed twice with $10 \mathrm{~mL}$ volumes of TBS. The final erythrocyte pellets of 12 tubes ( $21 \mathrm{~mL}$ settled volume of cells) were resuspended to $30 \mathrm{~mL}$ in TBS and stored at $-20^{\circ} \mathrm{C}$.

\section{Isolation of Hemoglobin}

Isolation of hemoglobins was done by osmotic or freeze thaw lysis of erythrocytes previously washed with Tris-buffered saline. Erythrocytes in $30 \mathrm{~mL}$ of TBS (21 $\mathrm{mL}$ settled volume) were thawed from minus $20^{\circ} \mathrm{C}$ storage. This treatment lysed the cells and released their contents. After centrifugation for $20 \mathrm{~min}$ at $14,000 \mathrm{rpm}$ $(>13,000 \mathrm{~g})$ in a Hettich Mikro 120 (in $1.5 \mathrm{~mL}$ volumes), the supernatants were retained and combined as a hemoglobin preparation

\section{Agarose gel Electrophoresis}

Agarose gel electrophoresis was done by using 2-2.5 $\mathrm{cm}$ thick $1 \%$ agarose gels in $25 \mathrm{mM}$ Tris $250 \mathrm{mM}$ Glycine pH 8.3 buffer. A Biorad Midi horizontal gel electrophoresis chamber was used. The thickness of the gels accommodated larger sample volumes to enhance visualization of the red color of hemoglobin and its complexes. Sample buffer consisted of $200 \mu \mathrm{L}$ of 250 $\mathrm{mM}$ Tris, $25 \mathrm{mM}$ glycine $\mathrm{pH} 8.3$ mixed with 800 microliters of water and $1 \mathrm{~mL}$ of glycerol.

Sample preparation for electrophoresis was done for samples with and without addition of plasma or purified haptoglobin. To $50 \mu \mathrm{L}$ of hemoglobin was added $20 \mu \mathrm{L}$ of sample buffer or $50 \mu \mathrm{L}$ of plasma or for negative controls - $50 \mu \mathrm{L}$ of Tris Glycine buffer. To assess interaction of hemoglobins from different animal species with human haptoglobin variants Hp1:1 and Hp $2: 2,50 \mu \mathrm{L}$ of $2 \mathrm{mgmL}$ of haptoglobin were mixed with $50 \mu \mathrm{L}$ of hemoglobin and $20 \mu \mathrm{L}$ of sample buffer.Fifty $\mu \mathrm{L}$ to seventy $\mu \mathrm{L}$ of samples of different hemoglobin from different animal species with or without plasma were loaded in the sample wells of gels which were run at $80-100 \mathrm{~V}$ at constant voltage until a bromophenol blue marker in one sample well was about one centimeter from the end of the gel.

In the case of experiments to assess the interaction of hemoglobins from different animal species with horse plasma, $10 \mu \mathrm{L}$ ofhemoglobin were mixed with $90 \mu \mathrm{L}$ of horse plasma and $10 \mu \mathrm{L}$ of sample buffer and $40 \mu \mathrm{L}$ of sample was loaded per sample well.In certain cases, the electrophoresis was photographically documented after the samples had moved 2 to $3 \mathrm{~cm}$ from the point of application to better visualize bands since they became fainter due to diffusion as electrophoresis progressed.

SDS polyacrylamide gel electrophoresis was performed according to the method of Laemmli (1970) and by using 7.5 or $4-20 \%$ gradient gels.

\section{Lateral Flow Immunoassay}

The Seratec Hemdirect lateral flow test from Serological Research was used to test immunoreactivity of hemoglobins with a monoclonal antibody to human hemoglobin. The manufacturer's instructions were followed. In brief, 5 drops of hemoglobin solutions from different animal species were added to the well of the lateral flow immunoassay device and allowed to flow along the strip and thus rehydrate colloidal gold particles coupled to one monoclonal antibody to hemoglobin test. The strip contained a test zone (T) immediately after the sample well that contains a second monoclonal antibody to human hemoglobin. If the hemoglobin also reacted with the second monoclonal antibody to hemoglobin the colloidal gold particles adhered to the test zone on the strip and formed a colored line. This lateral flow immunoassay contains a control zone (C)to the left of the test zone. This control zone contains antibodies to mouse immunoglobin and thus binds the colloidal goldanti-hemoglobin particles in the presence and absence of hemoglobin. It serves to indicate that the colloidal gold particles with anti-human hemoglobin are functional. A colored line in the test strip and the control strip indicated the presence of hemoglobin in the sample that was reactive with the anti-human hemoglobin.

\section{Preparation of Llama Hemoglobin-Sepharose CL- 4B Affinity Matrix}

Llama hemoglobin was covalently linked to cyanogen bromide-activated Sepharose CL-4B (CN Br- 
Sepharose) by basically following the manufacturer's protocol. In brief, llama hemoglobin was dialyzed for 32 $\mathrm{h}$ with three changes of buffer against $150 \mathrm{mM} \mathrm{NaCl}$, $20 \mathrm{mM} \mathrm{NaHCO} 3$ pH 8.3 buffer with three changes of buffer. The hemoglobin was diluted to $4 \mathrm{mgmL}$. Three grams of CN Br-Sepharose was rehydrated with $10 \mathrm{mM}$ $\mathrm{HCl}$ and washed with $400 \mathrm{~mL}$ of the same solution and then with $200 \mathrm{~mL}$ of $150 \mathrm{mM} \mathrm{NaCl}, 20 \mathrm{mM} \mathrm{NaHCO} 3 \mathrm{pH}$ 8.3 buffer in a sinter funnel. The washed and equilibrated $\mathrm{CN}$ Br-Sepharose was then mixed with $4 \mathrm{~mL}$ of $4 \mathrm{mgmL}$ Llama hemoglobin and incubated with mixing for $1 \mathrm{~h}$. at $20^{\circ} \mathrm{C}$. The Llama hemoglobin-Sepharose was washed with $100 \mathrm{~mL}$ of $25 \mathrm{mM}$ Tris $250 \mathrm{mM}$ glycine $\mathrm{pH} 8.3$ and finally stored in $15 \mathrm{~mL}$ of the same buffer at $4^{\circ} \mathrm{C}$.

\section{Llama Hemoglobin-Sepharose CL-4B} Chromatography

Llama hemoglobin covalently coupled to Sepharose CL-4B (llama $\mathrm{Hb}$-Sepharose) was used in batch affinity chromatography of llama, horse and human plasmas. Unmodified Sepharose CL-4B was used for as control. A volume of $0.8 \mathrm{~mL}$ of human plasma was incubated with $100 \mu \mathrm{L}$ of llama-Hb Sepharose and $4 \mathrm{~mL}$ of llama plasma or horse plasma were each incubated with 300 $\mu \mathrm{L}$ of llama-Hb-Sepaharose or control Sepharose at $4{ }^{\circ} \mathrm{C}$ for $32 \mathrm{~h}$. The llama-Hb-Sepaharose and Sepharoseincubations were washed twice by centrifugation with $1 \mathrm{~mL}$ of PBS for the incubations with human plasma and $2 \mathrm{~mL}$ of PBS for the incubations with llama and horse plasmas. The final pelleted llama $\mathrm{Hb}-$ Sepharose from the human plasma chromatography was resuspended to a final volume of $100 \mu \mathrm{L}$ with PBS and the pelleted llama $\mathrm{Hb}$-Sepharoses from the llama and horse plasmas chromatography were resuspended to a final volume of $300 \mu \mathrm{L}$ with PBS. The final washed llama $\mathrm{Hb}$-Sepharose and control Sepharose contained whatever proteins had bound after the incubation with the plasmas from various species. For analysis by SDS (sodium dodecyl sulfate) gel electrophoresis, the llama Hb-Sepharose CL-4B and unmodified Sepharose chromatography materials were mixed with an equal volume of SDS gel sample buffer and $30 \mu \mathrm{L}$ were loaded on gels.

\section{Results}

Differences and Similarities Among Hemoglobins from Different Species

Llama and other Non-Human Hemoglobins do not React with a Monoclonal Antibody to Human Hemoglobin in a Lateral Flow Immunoassay

A lateral flow immunoassay that used monoclonal antibodies to human hemoglobin was used to assess similarities and differences in immunochemical reactivity among hemoglobins from llama, anteater and human (Fig.3). Human hemoglobin bound to the monoclonal anti-hemoglobin antibodies but none of the other animals' hemoglobins bound.

\section{Llama and Alpaca Hemoglobins Differ from Hemoglobins from Other Animal Species in Agarose Gel Electrophoretic Mobility}

Agarose gel electrophoresis was done for hemoglobins from different species in order to compare them for differences that might affect their binding to non-native haptoglobins (Fig. 4). The gel was not stained for protein and the hemoglobins were detected by their visible intrinsic red color. Llama and alpaca hemoglobins displayed the slowest electrophoretic mobilities.

Hemoglobins from Different Animal Species Interact with Human Plasma in a Way Consistent with Haptoglobin Binding

An experiment was done to test if different animal hemoglobins could bind with human haptoglobin by using agarose gel electrophoresis (Fig. 5).A second fainter hemoglobin band was present in all samples in the presence but not in the absence of plasma (Fig. 5). The second band containing llama hemoglobin migrated more slowly than those of the other species second hemoglobins (dashed arrow, Fig. 5). Llama hemoglobin alone (arrowhead, Fig. 5) migrates towards the anode, opposite to the movement of other species hemoglobins in agarose gel electrophoresis.

\section{Different Animal Species Haptoglobins form Complexes with Different Mobilities with two Different Human Plasmas as Detected by Electrophoresis}

Hemoglobins from llama, dog, human and pig were mixed with a second sample of human plasma (plasma B) as well as plasma $\mathrm{A}$ and analyzed by agarose gel electrophoresis in order to further demonstrate that hemoglobins from different animal speciescould bind humanhaptoglobin. The results again showed faster moving hemoglobin bands in the presence of the plasmas but not in the negative controls which lacked plasma (Fig. 6). In the case of plasma B, two faster movinghemoglobin bands were seen in the presence of plasma (arrow and dash, Fig. 6) as opposed to the one faster band seen with hemoglobins mixed with plasma A. One of the hemoglobin bands seen with plasma B migrated similarly to the faster band seen with hemoglobins in the presence of plasma $A$, but the second plasma B fast hemoglobin bands migrated less. It was evident that one of the presumptive llama hemoglobin-haptoglobin bands migrated more slowly than those of the other species (arrowhead, Fig. 6). 

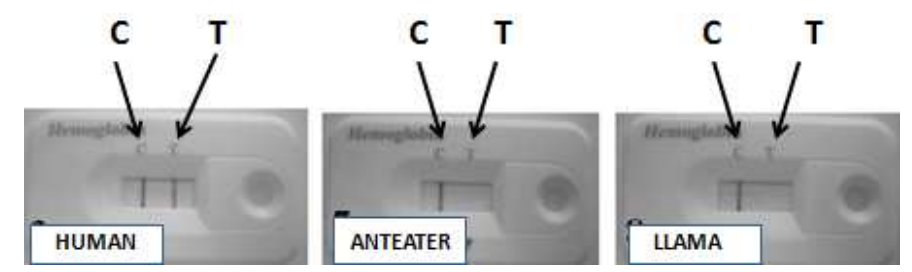

Fig. 3: Llama and anteater hemoglobins do not react with a monoclonal antibody to human hemoglobin in a lateral flow immunoassay: A lateral flow immunochromatographicassaybasedonmonoclonalantibodies to human hemoglobin was used to test hemoglobins from human, anteaterandllama. The presence of two lines in the assay device indicated a positive reaction for human hemoglobin and the presence of only one line indicated a lack of reaction for anteater and llama hemoglobins
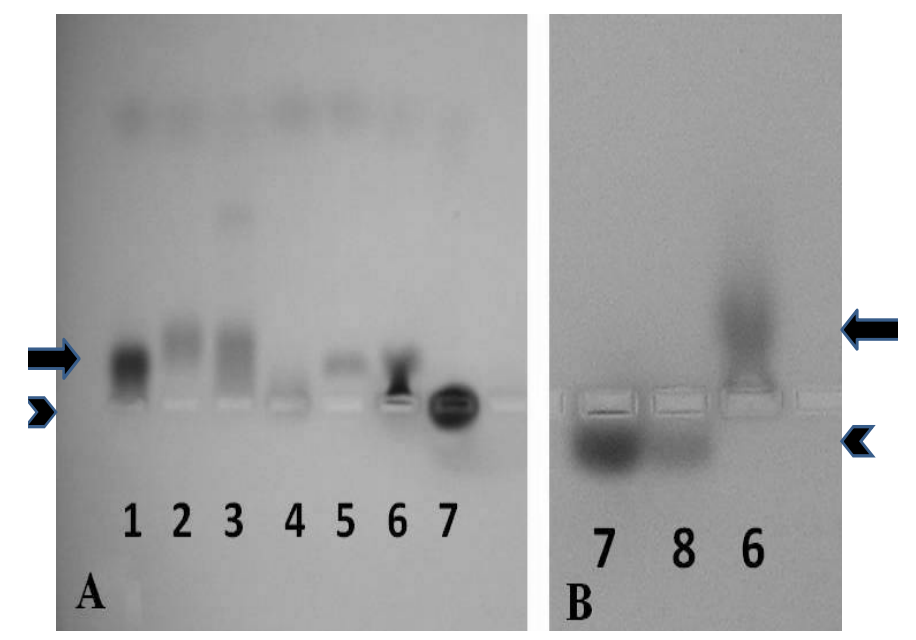

Fig. 4: Llama and alpaca hemoglobin differ from hemoglobins of other animals in mobility in agarose gel electrophoresis: Gel A. samples loaded from left are hemoglobins from: (1) Dog (2) Cat (3) Human (4) Anteater (5) Pig (6) Bovine (7) Llama. Gel B. samples from left are hemoglobins from: (7) Llama (8) Alpaca (6) Bovine. The cathode is at the top. Faint bands near the top of the gel in samples 1-6 are the bromophenol blue marker dye. Left arrow indicates positions of non-llama hemoglobins, left arrowhead indicates llama hemoglobin. Right arrow indicates position of bovine hemoglobin and right arrowhead indicates positions of llama and alpaca hemoglobins.

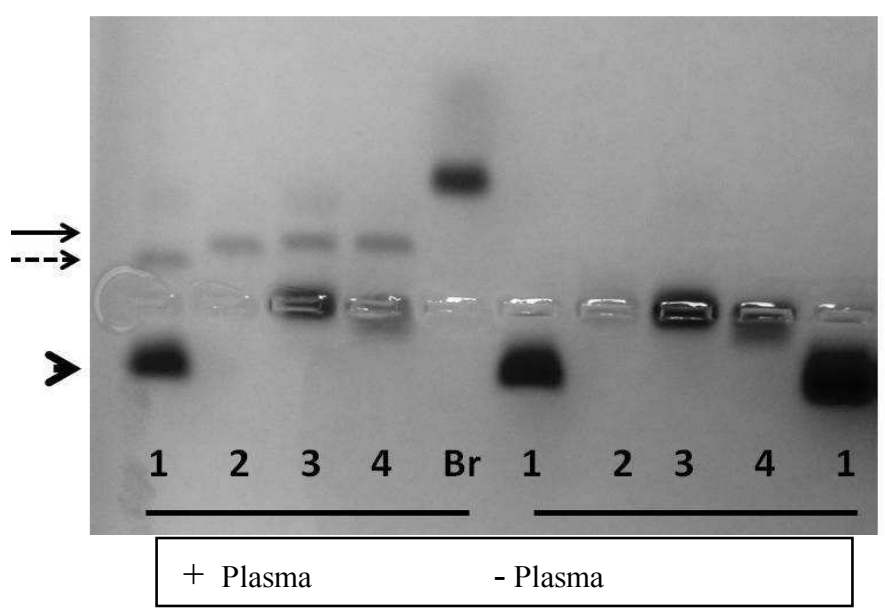

Fig. 5: Hemoglobins from different interact with human plasma in a way consistent with haptoglobin binding: Agarose gel electrophoresis was done with hemoglobin samples from different species in the presence $(+)$ and absence $(-)$ of single donor human plasma. From left the samples are hemoglobins from: (1) Llama (2) Human (3) Pig (4) Dog. Sample Br is the marker dye bromophenol blue alone. The solid arrow indicates the position of the second hemoglobin band in the presence of plasma in samples 2-4. The hatched arrow indicates the position of the second hemoglobin band in the presence of plasma for the llama sample. The arrow head indicates the position of the native (first) band of llama hemoglobin versus that of other species hemoglobins 


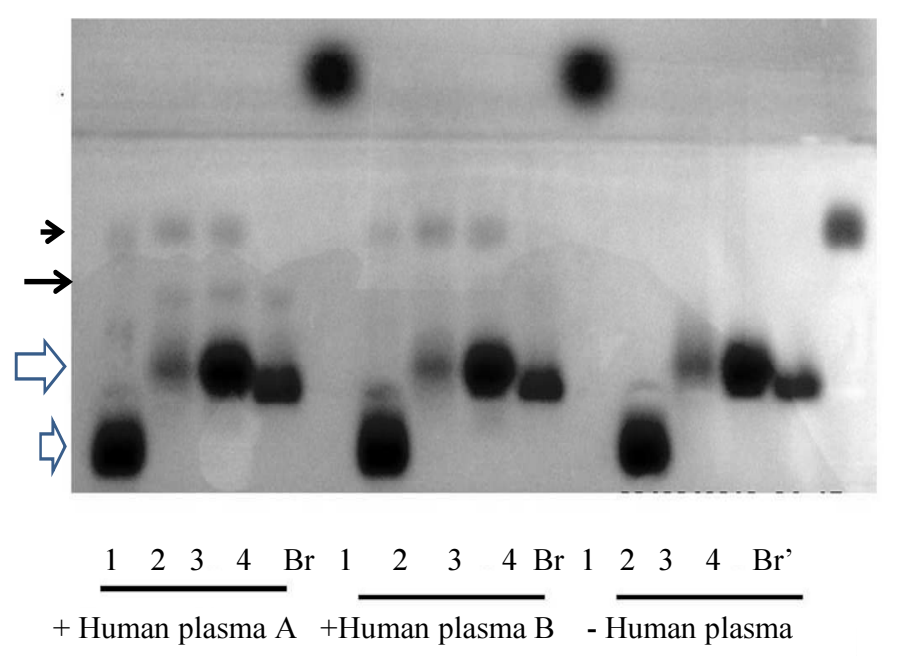

Fig. 6: Hemoglobins from different species form complexes with different mobilities with two different human plasmas as detected by electrophoresis: Agarose gel electrophoresis was done for hemoglobins from (1) Llama (2) Human (3) Pig (4) Dog in the presence of human plasma from donor A (first 4 samples) and donor B (next 4samples) and in the absence of plasma last 4 samples). The black arrowhead marks the position of the fastest moving of two additional hemoglobin-containing bands in the presence of plasma A. The black arrow on the left marks the position of a slower hemoglobin moving band in the presence of plasma A. The second llama hemoglobin band in the presence of plasma A migrates more slowly than those of other species. The open arrow indicates the positions of human, pig and dog hemoglobins and the open arrowhead indicates the position of llama hemoglobin. The last sample Br' is the bromophenol blue electrophoresis marker dye alone

\section{Different Animal Hemoglobins Interact with Horse Plasma in a Way Consistent with Haptoglobin Binding}

Llama, human, pig and dog hemoglobins all formed a second faster migrating band in the presence of horse plasma (Fig. 7). The faster moving hemoglobin in the presence of horse plasma moved at a similar position to the slower of the fast hemoglobin bands seen in the presence of human plasma B (dash, Fig. 7). The second faster moving hemoglobin band seen in the mixtures of different species hemoglobins with human plasma B had no counterpart in the hemoglobins mixed with horse plasma. It was also observed that the slower moving hemoglobin band in the llama sample (arrowhead, Fig. 7) migrated more slowly than the corresponding band formed with human, pig and dog hemoglobins. In the absence of plasma, no faster moving hemoglobin bands were seen.

\section{Different Animal Species Hemoglobins Bind to two Alleles of Human Haptoglobin (Non-Stained Gel: Early Stage of Electrophoresis)}

Hemoglobins from llama, pig, dog, goat, horse and human were mixed with purified genetic variants of human haptoglobin,Hp1:1 or $\mathrm{Hp} 2: 2$ and the mixtures were analyzed by agarose gel electrophoresis. The results for an early stage of electrophoresis are shown for the unstained gel in which the visualization of protein bands is by means of the intrinsic red color of hemoglobin (Fig. 8). The hemoglobins from llama, pig, dog, goat, horse and human formed a second faster migrating hemoglobin band with both haptoglobins $1: 1$ and $2: 2$.The same electrophoresis gel was allowed to run for alonger period of time so that proteins migrated further into the gel and was stained for protein with Coommassie Blue (Fig. 9). Two protein bands were seen in the hemoglobin samples that were mixed with haptoglobins. However the second fast moving band (arrow A) in samples mixed with haptoglobin 1:1 migrated further than those mixed with haptoglobin $2: 2$ (arrow A1). It was also the case that llama hemoglobin formed more slowly migrating complexes with both haptoglobin variants compared to the hemoglobins from other species. The position of free haptoglobin alone in the last gel lane is indicated by the thick arrowhead in Fig. 9. It was also evident that where hemoglobins were run in electrophoresis in the absence of haptoglobin, only one slow moving (human hemoglobin) or backwards moving (llama) hemoglobin was present.

\section{Llama Hemoglobin Binds Horse and Human Haptoglobin in Affinity Chromatography}

Affinity chromatography by using Llama hemoglobin that was covalently linked to Sepharose CL-4B was done to test for the binding ofhaptoglobin in plasmas ofllama, human and horse. As a negative control, Sepharose 4B was used in chromatography of samplesof the same plasmas. The samples that bound to llama hemoglobin-Sepharose and to plain Sepharose were analyzed by SDS gel electrophoresis under reducing conditions (Fig. 10). 


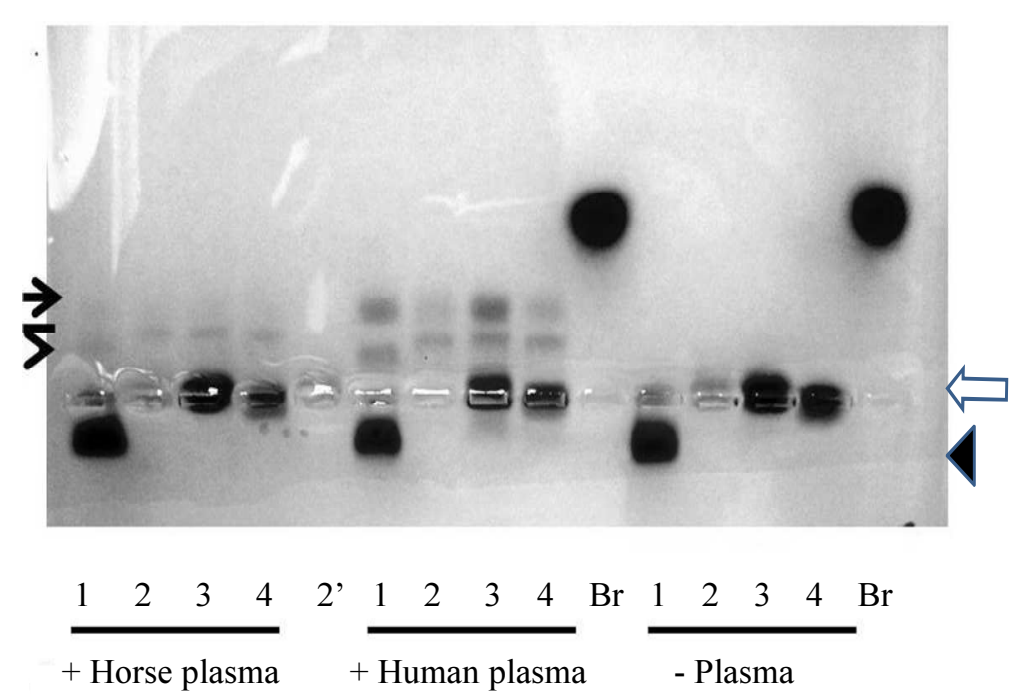

Fig. 7: Hemoglobins from different species interact with horse plasma in a way consistent with haptoglobin binding: Hemoglobin samples from (1) Llama (2) human (3) Pig (4) Dog were subjected to agarose gel electrophoresis in the presence of horse plasma (first 1-4) or the presence of human plasma donor B (second 1-4) or in the absence of plasma (last 1-4). The left dash marks the position of the second hemoglobin band seen in the presence of horse plasma. The left arrow marks the position of the furthest moving of the two bands seen in hemoglobins incubated with human plasma B. The left arrowhead marks the position of the second (slower moving) hemoglobin band seen in the presence of human plasma B. The fast moving hemoglobin band marked with a dash in samples mixed with horse plasma migrates in between the two faster moving hemoglobin bands seen in the presence of human plasma B. The right solid arrowhead marks the position of free llama hemoglobin and the right white arrow marks the positions of other animal hemoglobins

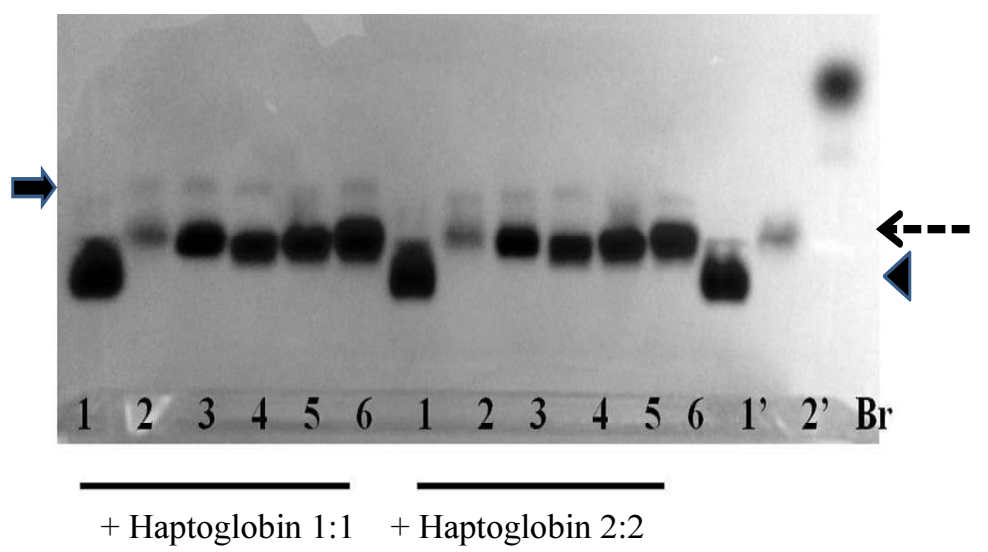

Fig. 8: Hemoglobins from different species bind two alleles of human haptoglobin: Non stained agarose gel in the early stage of electrophoresis of hemoglobins from (1) Llama (2) Human (3) Pig (4) dog (5) Goat (6) Horse in the presence of human haptoglobin 1:1 (first set of samples 1-6) or human haptoglobin 2:2 (second set of samples 1-6). Samples 1' and 2' are llama and human hemoglobin in the absence of haptoglobin and $\mathrm{Br}$ is the bromophenol blue marker alone. Left arrow indicates faster moving hemoglobin band. Right arrowhead and dashed arrow indicates positions of free llama hemoglobin and free hemoglobins from other species respectively

A protein of approximately $40 \mathrm{kDa}$ was isolated from both human and horse plasma but not llama plasma by llamahemoglobin Sepharose. This 40kDa protein was not present in the fraction bound by the negative control matrix Sepharose 4B alone but a 50kDA protein was present in the fraction retained from human plasma (Fig. 10). 


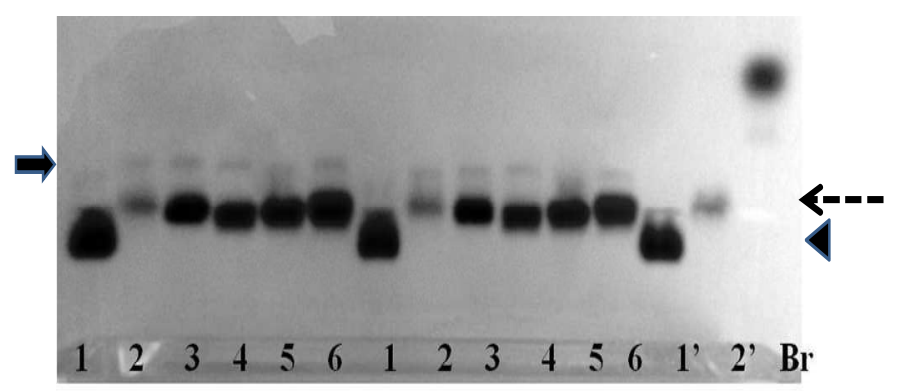

+ Haptoglobin 1:1 + Haptoglobin 2:2

Fig. 8: Hemoglobins from different species bind two alleles of human haptoglobin: Non stained agarose gel in the early stage of electrophoresis of hemoglobins from (1) Llama (2) Human (3) Pig (4) dog (5) Goat (6) Horse in the presence of human haptoglobin 1:1 (first set of samples 1-6) or human haptoglobin 2:2 (second set of samples 1-6). Samples 1' and 2' are llama and human hemoglobin in the absence of haptoglobin and $\mathrm{Br}$ is the bromophenol blue marker alone. Left arrow indicates faster moving hemoglobin band. Right arrowhead and dashed arrow indicates positions of free llama hemoglobin and free hemoglobins from other species respectively

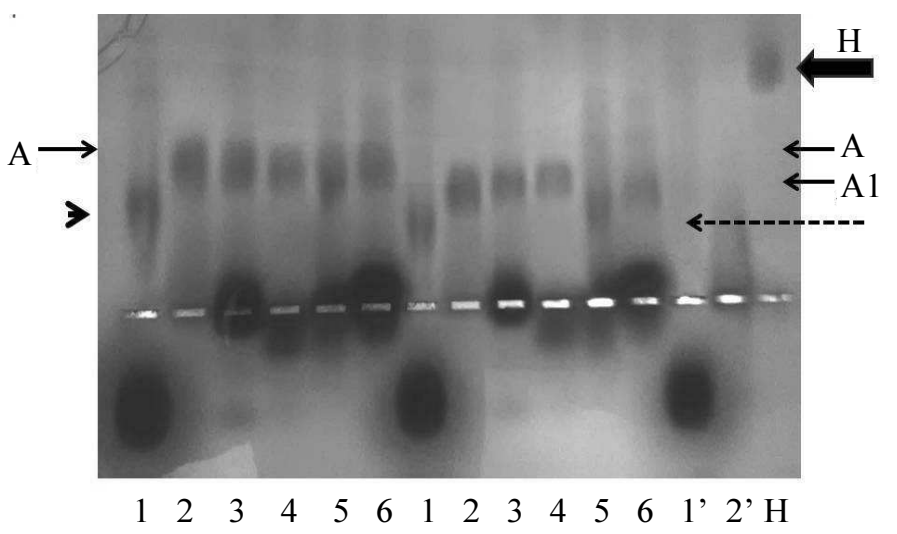

Fig. 9: Hemoglobins from different species bind two alleles of human haptoglobin Commassie blue-stained gel. The late stage of electrophoresis of hemoglobins from (1) Llama (2) Human (3) Pig (4) dog (5) Goat (6) Horse in the presence of human haptoglobin 1:1 (first set of samples 1-6) or human haptoglobin 2:2 (second set of samples 1-6). Samples 1' and 2' are llama and human hemoglobin in the absence of haptoglobin, $\mathrm{H}$ is haptoglobin $1: 1$ alone

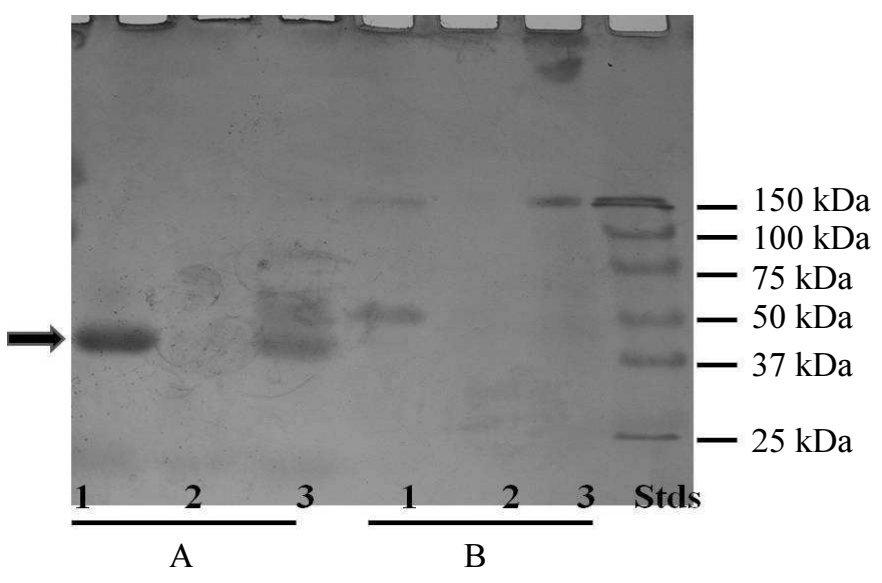

Fig. 10:Llama hemoglobin binds horse and human haptoglobin in affinity chromatography: An SDS gel shows samples retained by llama-hemoglobin Sepaharose (set A) and Sepharose 4B only (set B) from plasmas from (1) Human (2) llama (3) Horse. The arrow indicates a protein band present only in the fractions bound to llama-hemoglobin-Sepharose 


\section{Discussion}

This study supports the hypothesis that the ability of hemoglobins from different species to bind to haptoglobins of other species is conserved. The findings contradict the idea that evolutionary changes in hemoglobins and haptoglobins prevent cross species invitro interactions of these proteins. This study implies the occurrence of conserved protein-protein interactions sites on hemoglobin and haptoglobin.

The conserved binding hypothesis was examined by (1) showing that previously unreported differences exist among hemoglobins (2) testing the abilities of hemoglobins to bind haptoglobins of different species through electrophoresis of mixtures and affinity chromatography.

It was reported for the first time that llama and alpaca hemoglobins differed in electrophoretic mobility from hemoglobins from human, pig, dog, bovine, goat, horse, cat and anteater. The llama and alpaca hemoglobins had cathodal mobility and thus more positive charge than the other hemoglobins. The other animal hemoglobins also differed among each other in precise electrophoretic mobility indicating further subtle charge differences. This finding was also in accordance with the report that alpaca hemoglobin has only a single amino acid difference from that of llama (Piccinini et al., 1990).

A lateral flow immunoassay for human hemoglobin recognized hemoglobin from human but not hemoglobins from the other species tested including the previously unreported cases of llama and anteater. However, this assay recognizes certain non-human hemoglobins such as those of higher primates and ferrets. These results add to the documented differences and similarities in the hemoglobins from different animals (Perutz, 1983; Storz 2007; Ikehara et al., 1996; Lin et al., 1989). The immunochemical results indicate that a particular antigenic determinant on human hemoglobin was absent from hemoglobins from llama and the other animal species tested.

This study provided evidence that llama, goat, horse, dog, pig and bovine hemoglobins bind to human and horse haptoglobins because faster migrating hemoglobin bands were observed in the presence of different human plasmas, horse plasma and purified human haptoglobin variants. The formation of a second faster migrating band of hemoglobin after mixing of various animal hemoglobins with human and horse plasma is consistent with these hemoglobins binding to haptoglobin in these plasmas. Haptoglobin is considerably more acidic than hemoglobin so a complex of these proteins would migrate faster than hemoglobin but slower than haptoglobin.

The second hemoglobin band (haptoglobinhemoglobin complex), formed by hemoglobin from different species with horse plasma, migrated more slowly than the complex of hemoglobins with human haptoglobin (Hp 1:1) (Fig. 6 dash). This differentiates the horse from human haptoglobin.
It was also found that hemoglobins from different animal species bind to two purified human haptoglobin genetic variants. Non-human species do not express the polymorphic haptoglobin variants $\mathrm{Hp}$ 1:1 and $\mathrm{Hp}$ 2:2 that exist in humans (Liau et al., 2003; Wobeto et al., 2008) Binding of animal hemoglobins to these genetic variants of human haptoglobin such as by hemoglobins from goat, horse, pig, dog, bovine and llama further supports the hypothesis of conservation of binding sites on different species hemoglobins and haptoglobins. Purified haptoglobin variants $\mathrm{Hp} \quad$ 1:1 and $\mathrm{Hp}$ 2:2 incubated with hemoglobins from different species including human, formed only one faster mobility hemoglobin band for each haptoglobin variant (Fig. 9). The hemoglobin complexes with $\mathrm{Hp} \mathrm{1:1} \mathrm{had} \mathrm{faster}$ mobility than those with $\mathrm{Hp} 2: 2$. In contrast, in the case of hemoglobins incubated with plasma $B$, two new hemoglobin bands were observed. The different pattern is consistent with plasma B containing a third variant of haptoglobin $-\mathrm{Hp} 2: 1$.

\section{Conclusion}

It is therefore concluded that hemoglobin from the different animal species studied can bind to all three genetic variants of human haptoglobin.

Affinity chromatography of plasmas from different species on llama-hemoglobin-Sepharose (Fig. 10) also isolated a protein similar in molecular mass to haptoglobin from human and horse plasmas but not from llama plasma. Not all animal plasmas contain significant amounts of haptoglobin in the healthy state as for bovine and goat plasmas and this is thus indicated true for llama plasma.Human hemoglobin-Sepharose affinity chromatography has been previously used to isolate human haptoglobin (Liau et al., 2003) but llama hemoglobin has not previously been used in affinity chromatography.

In summary it is proposed that hemoglobinhaptoglobin binding sites were optimized early in evolution and afterwards retained for different species.

\section{Acknowledgement}

We are grateful for our students Christina Dunn and Netanya Kellam participating as undergraduate researchers during the Furman University Research Experience for Undergraduates (REU) 2016 session. They and Kicheryltus Troutman performed some supervised experiments. The REU was funded by an NSF grant NSF CHE-1460806 - awarded to K. Buchmueller and T. Hanks. This grant provided materials that allowed the further conduct of the research at Albany State University. Kicheryltus Troutman was supported by the Albany State University Center for Undergraduate Research which is funded by a Title III grant from the US Department of Education. 


\section{Author's Contributions}

Oluseyi A. Vanderpuye: Designed and performed experimnts, wrote the paper, analysed results.

Moges Woldemeskel: Conceived basis for research wrote sections of paper, checked and revised paper, evaluated experimental results.

\section{Ethics}

This study was done in compliance with applicable university regulations and rules for the use of human and animal commercial blood products, human and animal protection. All vendors comply with Federal and State laws.

\section{References}

Busby, W.H.andJ.C. Travis, 1978. Structure and evolution of artiodactyla haptoglobins. Physiol. Part B: Comp. Biochem., 60: 389-396. DOI: 10.1016/0305-0491(78)90066-4

Campbell, K.L.,J.E.E. Roberts, L.N. Watson, J. Stetefeld and A.M. Sloan et al., 2010. Substitutions in woolly mammoth hemoglobin confer biochemical properties adaptive for cold tolerance.Nature Genet., 42: 536-540. DOI:10.1038/ng.574

Cocca, E., M. Ratnayake-Lecamwasam, S.K. Parker, L. Camardella and M. Ciaramella et al., 1995. Genomic remnants of alpha-globin genes in the hemoglobinless antarctic icefishes. Proc. Natl. Acad. Sci. USA, 92: 1817-1821.

DOI: $10.1073 /$ pnas.92.6.1817

diPrisco, G., V. Carratore, E. Cocca, A. Riccio and M. Tamburrini, 2000. Molecular structure and functional adaptations of hemoglobins from Antarctic marine organisms. Ital. J. Zool. Suppl., 1: 37-46. DOI: 10.1080/11250000009356354

Godovac-Zimmermann, J., J. Kösters, G. Braunitzer and R. Göltenboth,1988. Structural adaptation of bird hemoglobins to high-altitude respiration and the primary sequences of black-headed gull (Larus ridibundus, Charadriiformes) $\alpha^{\mathrm{A}}-$ and $\beta / \beta^{\prime}-$ Chains.Biol. Chem. Hoppe Seyler., 369: 341-348. DOI: $10.1515 / \mathrm{bchm} 3.1988 .369 .1 .341$

Hiebl, I., R.E. Weber, D. Schneeganss, J. Kösters and G. Braunitzer,1988. High-altitude respiration of birds. Structural adaptations in the major and minor hemoglobin components of adult Rüppell's Griffon (Gyps rueppellii, Aegypiinae): A new molecular pattern for hypoxic tolerance. Biol. Chem. Hoppe Seyler., 369: 217-32.

DOI: $10.1515 / \mathrm{bchm} 3.1988 .369 .1 .217$

Ikehara, T., Y. Eguchi, S. Kayo and H. Takei,1996. Amino acid sequences of hemoglobin $\beta$ chains of five species of pinnipeds: Neophoca cinerea, Otaria byronia, Eumetopias jubatus, Pusa hispida and Pagophilus groenlandica. J. Protein Chem., 15: 659-65.

DOI: $10.1007 / \mathrm{BF} 01886748$
Jessen, T.H., R.E. Weber, G. Ferme, J. Tame and G. Braunitzer, 1991. Adaptation of bird hemoglobins to high altitudes: Demonstration of molecular mechanism by protein engineering. Proc. Natl. Acad. Sci. USA, 88: 6519-6522. DOI: $10.1073 /$ pnas.88.15.6519

Katnik, I., M. Pupek and T. Stefaniak, 1998. Cross reactivities among some mammalian haptoglobins studied by a monoclonal antibody. Comp. Biochem. Physiol. B Biochem. Mol. Biol., 119: 335-340. DOI: 10.1016/S0305-0491(97)00358-1

Kumazaki, T., N. Urushibara and S. Ishii, 1992. Amino acid sequence and disulfide-bridge location of canine haptoglobin. J. Biochem., 112: 11-19.

DOI: 10.1093/oxfordjournals.jbchem.a123847

Lai, I.H., K.Y. Lin, M. Larsson, M.C. Yang and C.H. Shiau et al., 2008. A unique tetrameric structure of deer plasma haptoglobin-an evolutionary advantage in the $\mathrm{Hp}$ 2-2 phenotype with homogeneous structure. FEBS J., 275: 981-93. DOI: $10.1111 / \mathrm{j} .1742-4658.2008 .06267 . \mathrm{x}$

Lai, Y.A., H.Y. Lai, C.F. Tseng, J. Lee and S.J.T. Mao, 2007. Evidence of tandem repeat and extra thiolgroups resulted in the polymeric formation of bovine haptoglobin: A unique structure of Hp 2-2 phenotype. J. Biochem. Mol. Biol., 40: 1028-1038. DOI: 10.5483/BMBRep.2007.40.6.1028

Laemmli, U.K., 1970. Cleavage of structural proteins during the assembly of the head of bacteriophage T4. Nature. 227: 680-685. DOI: 10.1038/227680a0

Liau, C.Y., T.M. Chang, J.P. Pan, W.L. Chen and J.T. Mao, 2003. Purification of human plasma haptoglobin by hemoglobin-affinity column chromatography. J. Chrom. B, 790: 209-216. DOI: $10.1016 / \mathrm{S} 1570-0232(03) 00128-4$

Lin, H.X., T. Kleinschmidt, M.L. Johnson and G. Braunitzer, 1989. Carnivora: The primary structure of the Pacific Walrus (Odobenus rosmarus divergens, Pinnipedia) hemoglobin. Biol. Chem. Hoppe Seyler., 370: 135-40. DOI: 10.1515/bchm3.1989.370.1.135

Mominoki, K., N. Nakagawa-Tosa,M. Morimatsu, B. Syuto and M. Saito, 1995. Haptoglobin in Carnivora: A unique molecular structure in bear, cat and dog haptoglobins. Comp. Biochem. Physiol. B Biochem. Mol. Biol., 110:785-9.

Niranjan, S.K., S. Goyal, P.K. Dubey, V. Vohra and K. Singh et al., 2015. Molecular characterization of buffalo haptoglobin: Sequence based structural comparison indicates convergent evolution between ruminants and human. J. Anim. Biotech. DOI: 10.1080/10495398.2015.1069302

Painet, B. and E. Jaenicke, 2010. Structure of the altitude adapted hemoglobin of guinea pig in the R2 state. PLoS One 5: e12389-e12389. DOI: 10.1371/journal.pone.0012389 
Perutz, M.F., 1983. Specific adaptations in a protein molecule. Mol. Biol. Evolut., 1: 1-28.

Piccinini, M., T. Kleinschmidt, K.D. Jürgens and G. Braunitzer, 1990. Primary structure and oxygenbinding properties of the hemoglobin from guanaco (Lama guanacoë, Tylopoda). Biol. Chem. Hoppe Seyler., 371: 641-8.

DOI: /10.1515/bchm3.1990.371.2.641

Quaye, I., 2008. Haptoglobin, inflammation and disease. Trans. R. Soc. Trop. Med. Hyg., 102: 735-742. DOI: 10.1016/j.trstmh.2008.04.010

Shaer, D.J., F. Vinchi, G. Ingoglia, E. Tolosanao and P.W. Buehler, 2014. Haptoglobin, hemopexin and related defense pathways - basic science, clinical perspectivesand drug development. Front. Physiol. DOI:10.3389/fphys.2014.00415

Stam, W.T., J.J. Beintema, R. D'Avino, M. Tamburrini and G. di Prisco, 1997. Molecular evolution of hemoglobins of antarctic fishes (Notothenioidei). J. Mol. Evol., 45: 437-437. DOI: 10.1007/PL00006248

Storz, J.F., 2007. Hemoglobin function and physiological adaptations to hypoxia in high altitude mammals. J. Mammal., 88: 24-31. DOI: 10.1644/06-MAMM-S-199R1.1

Storz, J.F. and H. Moriyama, 2008. Mechanism of hemoglobin adaptation to high altitude hypoxia. High Alt. Med. Biol. DOI:10.1089/ham.2007.1079
Tecles, F., S.M. Subiela, G. Petrucci, C.G. Panizo and J.J. Ceron, 2007. Validation of a commercially available immunoturbidometric assay for haptoglobin determination in canine serum samples. Vet. Res. Commun., 31: 23-26.

Watanabe, B., T. Maita, G. Matsuda, M. Goodman and M.L. Johnson, 1986. Amino-acid sequence of the $\alpha$ and $\beta$ chains of adult hemoglobin of the harbor seal, Phoca vitulina. Biol. Chem. Hoppe Seyler., 367: 1251-8. DOI: 10.1515/bchm3.1986.367.2.1251

Wicher, K.B. and E. Fries, 2006. Haptoglobin, a hemoglobin-binding plasma protein, is present in bony fish and mammals but not in frog and chicken. Proc. Natl. Acad. Sci. USA, 103: 4168-4173.

Wicher, K.B. and E. Fries, 2010. Evolutionary aspects of hemoglobin scavengers. Antioxid. Redox Signal., 12: 249-59. DOI: 10.1089/ars.2009.2760

Wicher, K.B. and E. Fries, 2007. Convergent evolution of human and bovine haptoglobin: Partial duplication of the genes. J. Mol. Evol., 65: 373-9.

Wobeto, V.P., T.R. Zaccarietto and M.F. Sonnati, 2008.Polymorphism of human haptoglobin and its clinical importance. Genet. Mol. Biol., 31: 602-620. 